\title{
Betwixt and between: a surgical post-acute treatment unit (SPA) for the optimal care of elderly patients with isolated hip fractures
}

\author{
Jody C. DiGiacomo ${ }^{1,2}$ (D) L. D. George Angus ${ }^{1,2} \cdot$ Sara Cardozo-Stolberg $^{1,2} \cdot$ Raina Wallace $^{2} \cdot$ Noam Gerber $^{1,2}$. \\ Swapna Munnangi ${ }^{1,2} \cdot$ Shyni Charley ${ }^{3} \cdot$ Karen McGlynn $^{3}$
}

Received: 21 September 2018 / Accepted: 3 January 2019 / Published online: 30 January 2019

(c) Springer Nature Switzerland AG 2019

\begin{abstract}
Background A performance improved project identified elderly trauma patients to have a disproportionate incidence of complications.

Aims The purpose of this study was to assess the efficacy of a small specialty care unit to decrease complications in patients who no longer warrant care in an intensive care unit (ICU).

Methods A surgical post-acute treatment unit (SPA) was developed with focused attention to cognition, nutrition, respiration, and mobilization needs of patients who no longer had physiologic need for an intensive care unit environment, but were still in need of increased attention at the bedside.

Results While ICU and hospital lengths of stay were unchanged, patients placed in the SPA experienced less complications and required less unplanned ICU re-admissions.

Discussion Geriatric patients, especially the elderly, are not simply old adults. They have unique needs as a consequence of the aging process, which can be encompassed by four pillars of intercession: cognition, nutrition, respiration, and mobilization. Conclusions By adapting a physical environment supported by bedside attention to address the interwoven needs of geriatric and elderly patients who no longer care in an ICU, complications and unplanned return admissions to the ICU can be decreased.

Level of evidence III.
\end{abstract}

Keywords Geriatric · Elderly · Trauma $\cdot$ Hip fracture $\cdot$ Complications

\section{Introduction}

The 2010 US Census reported that $13 \%$ of the US population is aged 65 years old and older and this proportion is expected to grow to $22 \%$ by 2020 [1]. In relation, geriatric trauma is increasing both in absolute numbers and relatively as a proportion of trauma patients presenting to trauma centers. It is currently the seventh leading cause of death for this population [2]. Geriatric trauma patients have been reported to have

Jody C. DiGiacomo

Jdigiac1@numc.edu

1 Division of Trauma, Nassau University Medical Center, 2201 Hempstead Turnpike, East Meadow, NY 11554, USA

2 Department of Surgery, Nassau University Medical Center, 2201 Hempstead Turnpike, East Meadow, NY 11554, USA

3 Department of Nursing, Nassau University Medical Center, 2201 Hempstead Turnpike, East Meadow, NY 11554, USA worse outcomes compared to non-geriatric trauma patients [3-5]. Identified factors which lead to worse outcomes in geriatric trauma patients include an increased need for more resource intensive care, increased comorbid medical conditions, medications and frailty. Frailty is an indicator of senescence and is clinically distinct from age, comorbidities, and disability. It reflects the decreased physiologic reserve across multiple organ systems, leading to an impaired ability to tolerate physiologic stress [6].

\section{Background}

In response to an unplanned Surgical Intensive Care Unit (SICU) admission in 2015 of a trauma patient who had an aspiration event, a focused Trauma Performance Improvement review was conducted for a 4-year period, 2012-2015. There were 23 unplanned SICU admissions 
over that 48-month period, of whom 7 patients died (Table 1). There was a marked increase in 2014 coincident with a $30 \%$ increase in trauma admissions. The 23 unplanned SICU admissions were characterized by patients of elderly age and of limited or impaired mobility who developed dehydration resulting in hypernatremia, respiratory complications and renal dysfunction. Previous research has identified a population of geriatric patients who have a significantly increased risk of dehydration due to exceptionally low intake of fluids. This population has impaired cognition and mobility due to dementia, injury, or pre-existing muscular-skeletal conditions and exhibits an inability to express a desire for drinks. As a result, they require more attention than that which is provided by the typical 1-to-8 nurse-to-patient ratio in order to identify low fluid intake and prevent dehydration. The etiology appeared to be the same as that identified by Doreen Norton, R. N. in 1962: "The exceptionally low intake.. . appears to have been due to a combination of these factors with one factor predominant- they could not, or did not, express a desire for drinks and their low intake was not recognized." As a result, they became dehydrated. Combined with their immobility, the dehydration also contributed to thickened respiratory secretions, which required more effort to clear at a time when they had difficulty to do so. They developed atelectasis, pulmonary consolidation, respiratory distress, and pneumonia [7]. We postulated that perhaps these elders had not received a degree of individual attention commensurate with their needs and they simply needed an increased level of support and attention at the bedside.

Based on detailed chart review of the unplanned ICU admissions of these elders, we identified four pillars upon which to build an intercession: cognition, nutrition, respiration, and mobilization, all of which were addressed with multiple interventions, many of which overlapped. All require the delivery of attention at the bedside.

The Surgical Post-Acute Treatment Unit (SPA) was developed to better meet the specialized considerations in patient care, interventions, and environment of this particular geriatric population, and a separate SPA registry maintained $[8,9]$. The SPA is a six-bed unit without cardiac monitoring adjacent to the Critical Care area, supported by one registered nurse and two patient care assistants (PCA) chosen specifically for their affinity for working with elderly patients. Clinical medical management is provided by the SICU attending and resident team in the SPA as it is in the SICU and overseen by the trauma program. The purpose of this study is to evaluate the effect that programmatic changes resulting in the creation of the Nassau University Medical Center's (NUMC) SPA has had on treatment outcomes in geriatric trauma patients.

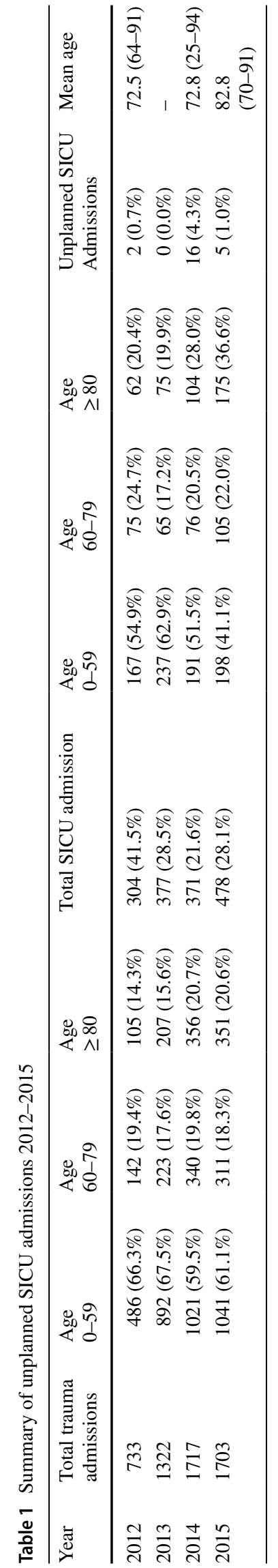




\section{Materials and methods}

Due to the myriad combinations of injuries and surgical diagnoses of patients admitted to the SPA, in an effort to reduce confounding variables, it was decided to perform our initial retrospective review of the impact of the SPA on an as homogeneous population as possible. Therefore, the injury of isolated hip fractures due to trauma was chosen. An IRB-approved retrospective review was performed on the specific subgroup of patients aged 70 and above with isolated hip fractures during the last 6 months of 2014, before the SPA was opened, and the last 6 months of 2016, when the SPA was fully operational. These patients were identified by review of the Trauma Registry and the separate SPA-specific registry, supplemented as needed by review of the electronic medical records. The historical control group (Pre-SPA) consisted of patients admitted from 1 July 2014 to 31 December 2014 and the study group (SPA) was patients admitted to the SPA between 1 July 2016 and 31 December 2016. A clinical pathway for the management of hip fractures was in place and unchanged throughout these time periods, the difference being the study group was admitted to the SPA from the ICU post-operatively instead of to a regular medical/surgical floor for the historical control group [10]. Data collected and analyzed on these two groups included standard demographic information, injury and diagnosis information, hospitalization information, and outcome information such as discharge disposition, overall hospital and subunit length of stay, and complications.

\section{Statistical analysis}

Univariate statistics were used to measure the distribution of the covariates in the study sample. Means and standard deviations were used for continuous variables. Absolute and relative frequencies (i.e., $n$ and \%) were used for categorical variables. Bivariate statistical tests were used to measure for differences in the distribution of the covariates, the study group and comparator group. Student's $t$ test was used on continuous covariates. Chi-square and fisher's exact tests were used on categorical covariates. All data management and analyses will be conducted using Microsoft excel and SAS.

\section{Results}

There were 117 patients in the pre-SPA group and 105 in the SPA group. The pre-SPA and SPA populations were compared and found to be comparable with regard to
Table 2 Demographic characteristics of pre-SPA and SPA patient populations

\begin{tabular}{llll}
\hline & Pre-SPA & SPA & $p$ \\
\hline$n$ & 117 & 105 & \\
Age & 83.3 & 85.0 & 0.08 \\
Male/female & $29 / 88$ & $24 / 81$ & 0.73 \\
MOI: fall & 111 & 105 & 0.11 \\
ISS & 10.1 & 9.2 & 0.31 \\
RTS & 7.76 & 7.82 & 0.32 \\
CHF & 9 & 17 & 0.06 \\
Dementia & 25 & 26 & 0.63 \\
Diabetes & 27 & 17 & 0.24 \\
Hypertension & 24 & 30 & 0.21 \\
Tobacco & 4 & 7 & 0.36 \\
\hline
\end{tabular}

No differences were statistically significant

MOI mechanism of injury, ISS Injury Severity Score, RTS revised trauma score, $\mathrm{CHF}$ congestive heart failure

demographics, mechanism of injury, co-morbid conditions, ISS, and RTS (Table 2).

The ICU length of stay (ICU-LOS) for the two groups was similar, being 3.9 days for the pre-SPA group and 2.8 days for the SPA group $(p=0.84)$. As it is has been our routine to admit all hip fracture patients directly to the SICU from the Emergency Department pre-operatively, this ICULOS includes the pre-operative and post-operative time in the SICU [10]. Similarly, the hospital length of stay (HLOS) and post-ICU length of stay were similar for the two groups.

As compared to the pre-SPA group, who were managed in a standard floor setting after surgery, the SPA group had statistically significant reductions in unplanned SICU admissions ( 8 vs. $1 ; p=0.04$ ); sepsis events ( 6 vs. $0 ; p=0$. 03 ); and urinary tract infections ( 13 vs. $0 ; p=0.0002$ ). The pre-SPA population of 117 patients experienced 7 deaths, while the SPA group of 105 patients had only $1(p=0.07)$ (Table 3).

There was one death in the SPA group of elders with isolated hip fractures: An 88-year-old woman who developed an ST-elevation myocardial infarction on her 4th post-operative day. She was transferred back to the SICU, intubated, and given thrombolytic therapy. Her family withdrew support 2 days later. She accounted for the sole events of myocardial infarction, unplanned admission to the ICU, unplanned intubation, and death in the SPA group.

\section{Discussion}

The concept that older patients constitute a population with unique characteristics and needs is not new. In 1946, the Representative Body of the British Medical Association 
Table 3 Outcomes pre- and post-creation of the SPA

\begin{tabular}{llll}
\hline & Pre-SPA & SPA & $p$ value \\
\hline$n$ & 117 & 105 & \\
ICU-LOS & 3.9 & 2.8 & 0.84 \\
HLOS & 5.9 & 5.2 & 0.52 \\
Deaths & 7 & 1 & 0.07 \\
Cardiac arrest & 4 & 0 & 0.12 \\
Myocardial infarction & 3 & 1 & 0.25 \\
Pneumonia & 2 & 0 & 0.50 \\
Pulmonary embolism & 2 & 0 & 0.50 \\
Sepsis & 6 & 0 & $\mathbf{0 . 0 3} *$ \\
Unplanned ICU Admission & 8 & 1 & $\mathbf{0 . 0 4} *$ \\
Unplanned intubation & 2 & 1 & 0.50 \\
UTI & 13 & 0 & $\mathbf{0 . 0 0 0 2} *$ \\
\hline
\end{tabular}

$I C U-L O S$ intensive care unit length of stay, HLOS hospital length of stay, UTI urinary tract infection

$p<0.05$ in bold with asterisk

adopted a resolution to investigate "the inadequate...treatment and care of the elderly", which resulted in the 1947 Report of the Committee on the Care and Treatment of the Elderly and Infirm [11]. They accurately predicted the near doubling of the number of individuals more than 60 years old and that their most common causes of serious physical illness would be cardiac, cerebro-vascular, and "rheumatic". They concluded, "It is essential that in selected general hospitals of the future, including teaching hospitals, there should be geriatric departments for the investigation and treatment of elderly patients."

The first supplement to that report a year later, entitled "The right patient in the right bed", advocated special accommodations "in the form of hostels...staffed by nursing orderlies and attendants with a minimum of skilled nursing supervision" so as too not occupy acute care beds by patients who did not require expert nursing [12]. In 1954, G. F. Adams described the advantages of rest homes for "so-called frail ambulant patients": An intermediate care facility for elderly patients whose needs were betwixt and between the acute care facility and recovery homes, whose purpose is the same as the recovery home rather than convalescence $[13,14]$.

Our concept for a special care unit for patients after the acute phase of care was further inspired in part by Cosin who described in 1956 tiered levels of care for "individuals of pensionable age" who "had overcome the pathologic processes sufficiently to meet a new situation" and no longer met criteria for the acute care facility [15]. He emphasized the importance of physical and occupational therapists to the success of the "annexes", and pointed out the ability to replace nursing staff with trained technical staff.
By the early 1960s progressive, or tiered, care in response to the geriatric patients' needs was well established in the form of rehabilitation centers, skilled nursing facilities, and nursing homes, and the benefits to the patients, acute care hospitals, and health care systems had become clear [16-18]. It was not until recently, however, with the current geriatric population explosion of the baby boomers that these concepts have been taken to the next level and been extended from facilities outside the acute care hospital to the discreet and multidisciplinary field of the care of the acutely injured within the acute care hospital. Many have recognized the special needs of the acutely inured geriatric and elderly patient and have developed administrative subunits to address their unique and multi-disciplinary needs. Mangram described a geriatric trauma service, "G-60", with decreased ED-LOS, time to OR, ICU-LOS, and HLOS, although their decreased mortality rate was not statistically significant [19, 20]. Others have also reported benefits of units focused on the elderly: in the Emergency Department; inpatient service; and the intensive care unit [21-23].

Implicit in these elder-focused units are the six risk factors for delirium identified by Inouye and Vidan [24, 25]: Cognitive impairment, sleep deprivation, immobility, visual impairment, hearing impairment, and dehydration. These risk factors also play a role in patients admitted to the floor requiring transfer to an ICU in an unplanned fashion. Rubano identified the need for mechanical ventilation as present in over half of all patients initially admitted to the floor who subsequently required transfer to the ICU in an unplanned fashion, pneumonia in 30\%, and renal failure in $7.6 \%$ [26]. Other risk factors for unplanned admission to the ICU were major abdominal surgery, neurosurgery, orthopedic surgery, and chest injury. By reviewing these risk factors for delirium and unplanned ICU admission in the context of our experience with unplanned ICU admissions, we distilled these factors into four pillars upon which to build an intercession: cognition, respiration, nutrition, and respiration.

\section{Cognition: behavioral}

Lawton described a hierarchy of behavioral complexity, with life maintenance behaviors such as breathing being the lowest [27]. The next lowest level is functional health, or those behaviors associated with being bed bound or hospitalized, followed by perception/cognition; physical selfmaintenance; and then effectance, which White described as an inherent drive to interact with one's environment [28]. The goal of the behavioral interventions is to encourage the patient to move up this behavioral hierarchy to their premorbid levels of effectance. These older concepts have been subsumed by the current model of working memory, which is a short-term memory system involved in tasks that require concurrent retention and processing. It is composed 
of phonological and visuospatial components slaved to a central executive attention controller which allocates the limited working memory resources [29, 30]. The efficacy of the working memory normally deteriorates with aging [31, 32]. Executive function is further compromised by depression and the presence of cardiovascular disease [33, 34]. Physical activity, such as aerobic exercise and walking, has a salutary effect and improves executive function $[35,36]$.

\section{Cognition: environment}

In developing the concept of a post-acute care unit, it was important that the environment itself represented and reflected our desire that it be one that not only initiates the beginning of the physical recovery after the acute phase of injury and treatment, but also promotes a positive mental outlook toward health, recovery, and well-being by incorporating the approach and recommendations of Dahlberg and Olausson [8, 37-40]. By viewing the SPA not only as a physical place with concrete therapeutic goals but also as a transcendental experience for the patient and their family after the existential crisis of critical illness [41], this patientcentered focus allowed us to recognize the interplay between the physical environment of the SPA, the intercession pillar of cognition, and promoting the patient's positive attitude toward their recovery and their future thereafter. As an individual reaches the advanced ages where intellectual competence decreases, the proportion of behavior attributable to environmental cues and characteristics increases [27].

\section{Color}

Regardless of an individual's cultural background, the color blue and its harmonic color of green tend to be preferred [42, 43]. People associate blue with openness, peace, tranquility, and the ocean and sky, and environments rich in blues have been shown to enhance the performance of creative tasks [44, 45]. Unfortunately, blue has a low representation in cone sensitivity and the development of cataracts as part of the normal aging process makes these colors more difficult to see $[42,43,46]$. The walls and patient areas of the SPA were, therefore, painted sky blue for the upper two-thirds and a pastel green for the lower third to simulate a sense of openness and outside. Artwork-depicted arboreal scenery was placed on the walls for visual variety.

\section{Lighting}

Lighting and color are significant environmental attributes, especially for older patients [27]. Florence Nightingale recognized sunlight as essential to physical healing and mental well-being for reasons that have only been recently identified [47]. The importance of light and color continues to be recognized to promote physical and mental health in the elderly and can be used to address sleep disorders, depression, and behavioral issues in elders with dementia [27, 48-56].

It appears that these effects are specifically due to the presence of blue wavelength light. Intrinsically photosensitive retinal ganglion cells (ipRGCs) are blue wavelength light photoreceptors distinct from rods and cones. Rod cells mediate low light black and white vision. The three different types of cone cells respond to short wavelength (blue: $420-440 \mathrm{~nm}$ ), medium wavelength (green: 534-545 nm) or long wavelength (red: $564-580 \mathrm{~nm}$ ). ipRGCs have a distinct peak sensitivity to a range of $459-485 \mathrm{~nm}$, between the short and medium wavelength cones [57]. These cells connect to the suprachiasmatic nuclei ( $\mathrm{SCN}$ ) of the hypothalamus to influence the wake-sleep cycle and stimulate a near global increase in brain responsiveness for cognitive processes [45, 57-59].

The aging process compromises the reception and influence of blue light in a number of ways, especially after the age of 60 [46]. The aging brain has a decreased intrinsic cognitive responsiveness to blue light as compared to younger brains [60]. Senile miosis results in a narrowing of pupil diameter at all light irradiances and is more pronounced at lower light intensity (lux) [61]. In addition, as the lens itself ages, it yellows and opacifies (cataract) further decreasing the amount of light that falls on the retina, as well as filtering out increasing amounts of the $450 \mathrm{~nm}$ wavelength light to which the ipRGCs respond [42, 61-63].

It has long been recognized that fluorescent lighting is a poor substitute for natural light, causing headaches and eyestrain for some, and having a negative effect of work performance and mood due to its luminous and chromatic flicker and spectral distribution, especially below $525 \mathrm{~nm}$ [64-66]. The illumination of fluorescent lighting is also low, typically 50-300 lx in the hospital setting, and up to only 500-600 lx at midday in a typical patient room [67, 68]. Light of higher intensity (lux) and of higher temperature has been shown to be stimulating and pleasant, whereas light of lower lux and temperature is relaxing and cozy $[49,69,70]$.

The fluorescent lighting in the SPA was replaced with dual LED lighting: The baseline $3000 \mathrm{~K} 1500 \mathrm{~lm}$ lamps remain on at all times supplemented by $5000 \mathrm{~K} 1650 \mathrm{~lm}$ lamps from 6 a.m. to 9 p.m. The 3000 K LED lamps have a peak output broadly centered at $600 \mathrm{~nm}$, the yellow-orange spectral range, with little blue output and are of lower luminous flux, resulting in warm yellow white illumination [71, 72]. The 5000K LED lamps have a peak output centered at $450 \mathrm{~nm}$ and high luminous flux, resulting in a bright white illumination to stimulate the SCN both through the high illumination and the blue light MRG cell-mediated pathways. This cycling of high temperature high lux light rich in blue light wavelengths with lower temperature low lux relatively 

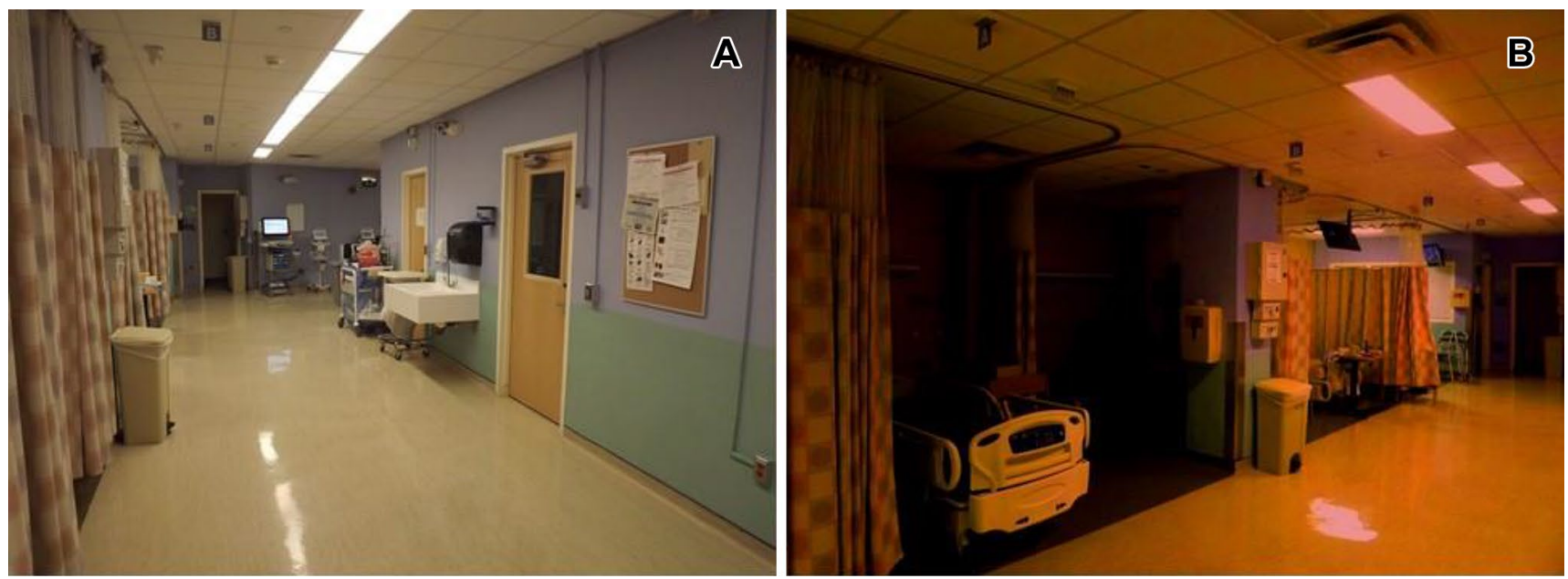

Fig. 1 SPA Lighting. a Daytime, b Nighttime

blue wavelength depleted light entrains the circadian rhythm, improves sleep, and reduces delirium [73-77] (Fig. 1).

\section{Noise}

Florence Nightingale recognized in 1859: "Unnecessary noise is the most cruel abuse of care which can be inflicted on either the sick or the well." [47] The movement of carts or the clicking of hard heels in the hallways, cardiac monitors, overhead pages, medical equipment alarms, and even the voices of staff have all been identified as specifically problematic $[78,79]$. The decision was made to not have cardiac monitors or continuous pulse oximetry in the SPA, and to cap intravenous catheters instead of continuous administration of intravenous fluids, even if at only a keep-vein-open rate. This not only reduces the ambient noise, but also avoids the patients' physical connections to wires and the equipment associated with illness, as well as the psychological connection to the process of illness, to optimize the cognitive behavioral paradigm of recovery, improvement, and wellness. In addition, the SPA ceiling mounted speakers had their volumes significantly reduced, and turned off altogether in the evening and overnight.

\section{Sleep}

The inability of the patient to sleep at night is a particularly vexing problem throughout any acute care facility. Sleep deprivation compromises the patient's cognition, promotes delirium, and will compromise their willingness to participate in physical therapy and other efforts of mobilization. The combination of the changes in lighting and noise reduction has been shown to improve sleep, decrease delirium, and decrease the need for hypnotics $[24,25,80]$.

\section{Nutrition}

Although many factors contribute to an individual's nutritional status, being of advanced age is associated with a poor nutritional state. Between 16 and $26 \%$ of older individuals who live alone suffer from malnutrition [81-83]. In the presence of pre-existing dementia, the incidence of malnutrition increases to 50\% [84]. While disabilities and frailty contribute to this, they are also consequences and are only exacerbated by the isolation of living alone, loneliness, and depression [84]. Other contributing factors include medical conditions as well as the medications prescribed to address them, which may have the side effects of dry mouth and loss of appetite [85]. The consequences of malnutrition are not only loss of nutritional reserves and weight loss due to the loss of muscle mass and body fat, and sarcopenia, but also anemia and the development of frailty [86-88]. Compromised cognitive function due to dementia, depression, and even the normal deterioration of working memory and executive function with aging has additive impacts, resulting in decreased caloric intake and inadequate fluid intake. In the inpatient setting, these issues may be further exacerbated. And if, as identified by Norton, the decreased intake goes unnoticed, the consequences can be dire [7].

To offset these issues, all patients transferred to the SPA must be able to take nutrition orally or via an enteral route. Each patient's intake is monitored. Nutritionists and speech pathologists assess every patient and formulate a customized nutrition plan which is implemented by the SPA staff and overseen by the entire treatment team. Recognizing that meal time is also an opportunity for social interaction and cognitive engagement, patients are attended during their meals and assisted if necessary by the SPA RN and/or PCAs. Families are encouraged to visit during mealtime and to bring in their loved one's favorite foods. 


\section{Respiration}

The high frequency of pulmonary compromise and complications was identified at our initial review of unplanned SICU admissions. Among elderly trauma patients in general, rib fractures rank third behind hip fractures and upper extremity fractures in frequency, being present in roughly $10 \%$ of patients with ground level falls [89]. It has been previously demonstrated that rib fracture protocols improve outcomes for these patients [90-93]. Review of successful interventions in the literature demonstrated one common pattern: increased attention to the patient at the bedside. The essential role of respiratory therapy and pulmonary toilette is the core of this pillar. The interventions of direct bedside care, getting out of bed to the chair, incentive spirometry, chest physiotherapy, and deep breathing and coughing are supplemented by the work of performing physical therapy.

\section{Mobilization}

The pillar of mobilization is entirely interwoven with the pillars of cognition, nutrition, and respiration. Low to moderate intensity activities improves cognitive function, physical functional capacity and quality of life [94-98]. Getting the patient out of bed into a chair, and daily physical therapy in the SPA are the two major components of this pillar. Physical therapy in our institution is provided at the bedside in the intensive care units, SPA, and medical/surgical wards based on the abilities and status of the individual patient, up to approximately $30 \mathrm{~min}$ a day, by dedicated physical therapists.

Elderly patients are well recognized to be at increased risks for complications after hip fracture surgery, with respiratory issues and urinary tract infections being the most frequent $[99,100]$. While the pre-SPA and SPA patient groups with isolated hip fractures were similar with regard to age, co-morbidities, ICU-LOS and HLOS, those patients who were admitted to the SPA enjoyed statistically significant decreases in the frequency of episodes of sepsis, urinary tract infections, and unplanned ICU admissions. Deaths decreased from $6.0 \%$ for pre-SPA patients (7/117) to $0.95 \%$ for SPA patients $(1 / 105)$. This approached but did not reach statistical significance due to the limited number of patients in this retrospective review. We interpret these improvements as consequences of the four pillars of intercession which constitute the foundation of the unique care environment in the SPA.

The SPA is associated with a small increased in cost as compared to the medical/surgical ward, but decreased cost as compared to the SICU. Nurse and PCA salaries are similar throughout our institution and are based on experience and longevity, so the differences in cost are due to the staffing of each unit. The SICU is a 10-bed unit staffed by 5 nurses and one PCA, and the medical/ surgical wards are 31 bed units staffed by 4 nurses and 3 PCAs. The SPA is a 6-bed unit staffed by one nurse and two PCAs. However, as previously discussed, the SPA is designed to attend to the needs of a population at increased risk for complications, and it is the decreased incidence of complications in these patients that realizes cost savings. The cost associated with a hospital acquired infectious complication in a non-operative patient population ranges has been estimated to range from $\$ 6542-10,375$ [101-103]. Even a urinary tract infection can add nearly $\$ 3000$ to the cost of an admission [104]. In a post-operative population, these additional costs can range from $\$ 8373$ for the delirium to over $\$ 50,000$ for a post-operative pneumonia requiring ventilator support [105-107]. For the SPA population, there were decreased complications of all types, with episodes of sepsis decreasing from 6 to zero $(p=0.03)$, urinary tract infections decreasing from 13 to zero $(p=0.0002)$, and unplanned ICU admissions decreasing from 8 to one $(p=0.04)$. We believe the cost savings through the decreased incidence of complications more than cover the modest increase of staffing in the SPA as compared to the medical/surgical ward.

This retrospective review of the impact of the SPA on the outcomes of elderly patients with isolated hip fractures has limitations. First, the number of patients is limited and further analysis of larger numbers is warranted to confirm the findings in this preliminary report. Second, only patients with isolated hip fractures were reviewed. Additional similar reviews of other isolated injuries and multi-system injuries are necessary, and will be the subject of subsequent analyses.

\section{Conclusion}

Providing improved and specialized care for our elderly in an organized fashion has been evolving since the 1940s, from rest homes for patients whose needs were "betwixt and between" the hospital and home, to multidisciplinary geriatric emergency departments, geriatric intensive care units, and geriatric in-patient services. We view our SPA as the next logical progression, a place betwixt and between the Surgical Intensive Care Unit and the standard floor setting for those patients who no longer need intensive medical care, but are not quite ready for the relative independence of the floor (Fig. 2). We believe it is important to recognize that the elderly are not simply older adults. They have unique physiology and needs. The SPA addresses those areas of need as four broad categories: cognition, nutrition, respiration, and mobilization. As a result, complications are decreased and outcomes are improved. 
Fig. 2 The SPA: a place betwixt and between

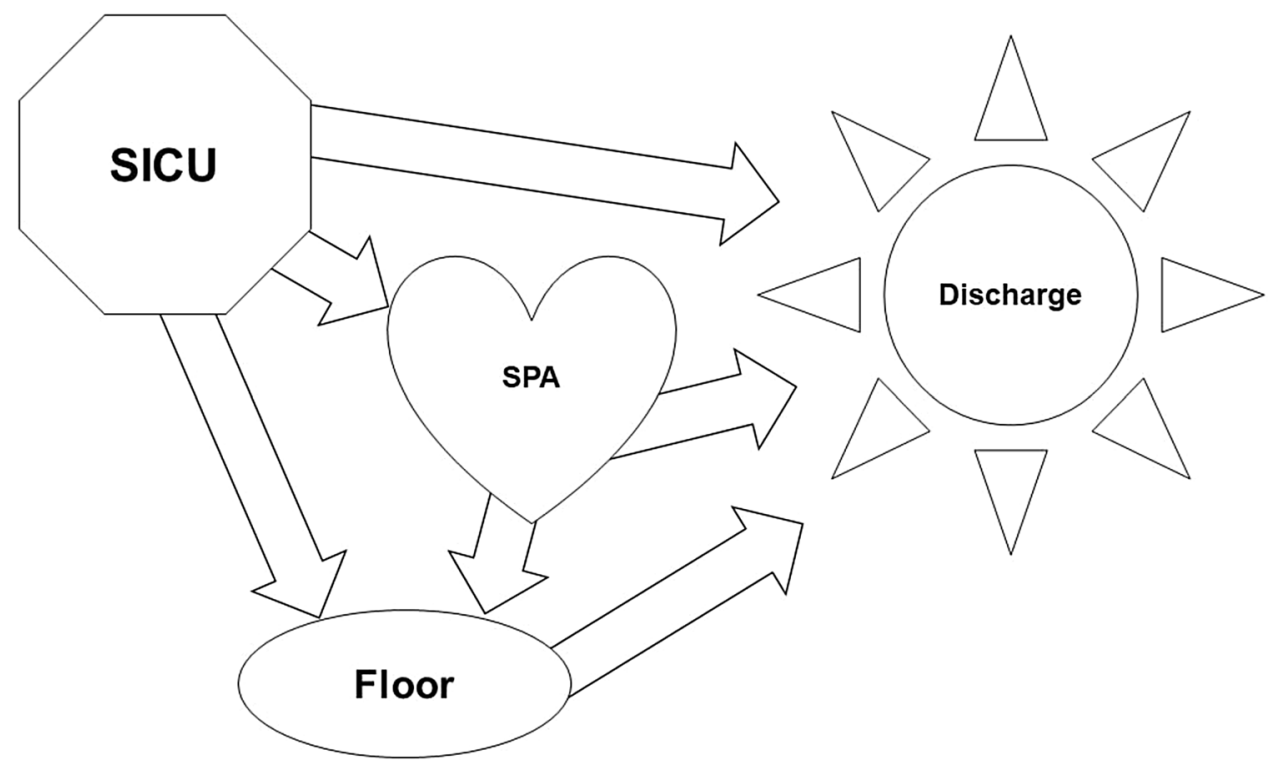

Author contributions JD: Study concept and design; acquisition of data; analysis and interpretation of data; drafting of manuscript; critical revision of manuscript. LDGA: Study concept and design; acquisition of data; analysis and interpretation of data; critical revision of manuscript. SCS: Study concept and design; critical revision of manuscript. RW: Acquisition of data; analysis and interpretation of data, drafting of manuscript. NG: Acquisition of data; analysis and interpretation of data, drafting of manuscript. SM: Acquisition of data; analysis and interpretation of data, drafting of manuscript. SC: Study concept and design; critical revision of manuscript. KM: Study concept and design; critical revision of manuscript.

Funding No funding was received.

\section{Compliance with ethical standards}

Conflict of interest Dr. DiGiacomo declares that he has no conflict of interests. Dr. Angus declares the he has no conflict of interests. Ms. Cardozo-Stolberg declares that she has no conflict of interests. Dr. Wallace declares that she has no conflict of interests. Mr. Gerber declares that he has no conflict of interests. Dr. Munnangi declares that she has no conflict of interests. Ms. Charley declares that she has no conflict of interests. Ms. McGlynn declares that she has no conflict of interests.

Ethical approval This project was IRB approved as exempt.

Statement of human and animal rights This article represents a retrospective review, approved by the Institutional Review Board of Nassau University Medical Center, East Meadow, New York.

Informed consent Informed consent was not required as this project was a retrospective review.

Availability of Data and Material De-identified data and material can be provided upon request.

\section{References}

1. United States Population Projections: 2000 to 2050. http:// www.census.gov/population/projections/files/analytical-docum ent09.pdf

2. Centers for Disease Control and Prevention: Injury prevention and control: data and statistics. http://www.cdc.gov/injur y/wisqars/pdf/leadingcausesofdeathbyagegroup2012-a.pdf

3. Davidson GH, Hamlat CA, Rivara FP et al (2011) Long term survival of adult trauma patients. J Am Med Assoc 305:10011007. https://doi.org/10.1001/jama.2011.259

4. Labib N, Nouh T, Wincour S et al (2011) Severely injured geriatric population. J Trauma 71:1908-1914. https://doi. org/10.1097/TA.0b013e31820989ed

5. Bergeron E, Clement J, Lavoie A et al (2006) A simple fall in the elderly: not so simple. J Trauma 60:268-273

6. Clegg A, Trust DM (2011) The frailty syndrome. Clin Med 11:72-75

7. Norton D, McLaren R, Exton-Smith AN (1962) Geriatric nursing problems in hospital. The National Corporation for the Care of Old People, London

8. Olausson S, Engwall M, Johansson L (2012) Evidence-based and sustainable design in a high tech hospital environmenta challenge for the future. Chalmers Conferences, ARCH12, Gothenburg, Sweden, 14-15 November 2012. http://hdl.handl e.net/2320/11837. Accessed 25 Jan 2019

9. Sommers J, Engelbert RH, Dettling-Ihnenfeldt D et al (2015) Physiotherapy in the intensive care unit: an evidence-based, expert driven, practical statement and rehabilitation recommendations. Clin Rehabil 29:1051-1063

10. Wallace R, Angus LDG, Munnangi S et al (2018) Improved outcomes following implementation of a multidisciplinary care pathway for elderly hip fractures. Aging Clin Exp Res. https:// doi.org/10.1007/s40520-018-0952-7 
11. British Medical Association (1947) Report of the committee on the care and treatment of the elderly and infirm. BMJ 1S:133-140

12. British Medical Association (1948) The right patient in the right bed. BMJ 2S:2272-2273

13. Adams GF (1954) Betwixt and between: a recovery home for the old. Lancet 264:486-488

14. British Medical Association (1955) Report of the geriatrics subcommittee. BMJ 1S:181-190

15. Cosin LZ (1956) A new approach to the problems of geriatric care. Kaiser Found Med Bull 4:321-328

16. Porter KRD (1962) Ward design. Lancet 2:35-37

17. Exton-Smith AN (1962) Progressive patient care in geriatrics. Lancet 1:260-263

18. Irvine RE (1963) Progressive patient care in the geriatric unit. Postgrad Med J 39:401-407

19. Mangram AJ, Mitchell CD, Shifflette VK et al (2012) Geriatric trauma service: a one-year experience. J Trauma 72:119-122

20. Mangram AJ, Shifflette VK, Mitchell CD et al (2011) The creation of a geriatric trauma unit "G-60". Am Surg 77:1144-1146

21. Geriatric Emergency Department Guidelines Taskforce (2014) Geriatric emergency department guidelines. Ann Emerg Med 63:e7-e25

22. Matzen LE, Jepsen DB, Ryg J et al (2012) Functional level at admission is a predictor of survival in older patients admitted to an acute geriatric unit. BMC Geriatr 12:32

23. Zeng A, Song X, Dong J et al (2015) Mortality in relation to frailty in patients admitted to a specialized geriatric intensive care unit. J Gerontol A Biol Sci Med Sci 70:1586-1594

24. Inouye SK, Bogardus ST, Charpentier PA et al (1999) A multicomponent intervention to prevent delirium in hospitalized older patients. N Engl J Med 340:669-676

25. Vidán MT, Sánchez E, Alonso M et al (2009) An intervention integrated into daily clinical practice reduces the incidence of delirium during hospitalization in elderly patients. J Am Geriatr Soc 57:2029-2036

26. Rubano JA, Vosswinkel JA, McCormack JE et al (2016) Unplanned intensive care unit admission following trauma. J Crit Care 33:174-179

27. Lawton MP (1970) Assessment, integration, and environments for older people. Gerontologist 10:38-46

28. White RW (1959) Motivation reconsidered: the concept of competence. Psychol Rev 66:297-333

29. Baddeley AD (1986) Working memory. Clarendon Press, Oxford

30. Baddeley AD, Hitch GJ (1974) Working memory. In: Bower GH (ed) The psychology of learning and motivation: advances in research and theory. Academic Press, New York

31. Kirova AM, Bays RB, Lagalwar S (2015) Working memory and executive function decline across normal aging, mild cognitive impairment, and Alzheimer's Disease. Biomed Res Int 2015:748212

32. Clark LR, Schiehser DM, Weissberger GH et al (2012) Specific measures of executive function predict cognitive decline in older adults. J Int Neuropsychol Soc 18:118-127

33. Liu ME, Chang YH, Ku YC et al (2012) Executive functions in elderly men. Age 34:59-66

34. Lockwood KA, Alexopoulos GS, van Gorp WG (2002) Executive dysfunction in geriatric depression. Am J Psychiatry 159:119-1126

35. Baker LD, Frank LL, Foster-Schubert K et al (2010) Effects of aerobic exercise on mild cognitive impairment: a controlled trial. Arch Neurol 67:71-79

36. Scherder EJA, van Paasschen J, Deijen JB et al (2005) Physical activity and executive functions in the elderly with mild cognitive impairment. Aging Mental Health 9:272-280
37. Dahlberg K, Drew N, Nystrom M (2001) Reflective lifeworld research. Studentlitteratur AB. Lund, Sweden

38. Dahlberg K (2006) The essence of essences-the search for meaning structures in phenomenological analysis of lifeworld phenomena. Int J Qual Stud Health Well-Being 1:11-19

39. Olausson S, Ekegergh M, Lindahl (2012) The ICU patient room: views and meanings as experienced by the next of kin: a phenomenological hermeneutical study. Intensive Crit Care Nurs 28:176-184

40. Olausson S, Lindahl B, Ekegergh M (2013) A phenomenological study of experiences of being cared for in a critical care setting: the meanings of the patient room as a place of care. Intensive Crit Care Nurs 29:234-243

41. Frank AW (1995) The wounded storyteller: body, illness, and ethics. University of Chicago, Chicago

42. Wijk H, Berg S, Sivik L et al (1999) Color discrimination, color naming and color preferences in 80-year olds. Aging Clin Exp Res 11:176-185

43. Dalke H, Littlefair PJ, Loe DL et al (2004) Lighting and colour for hospital design. The Stationary Office, Norwich

44. Kaya N, Epps HH (2004) Relationship between color and emotion: a study of college students. Coll Stud J 38:396-405

45. Mehta R, Zhu R (2009) Blue or red? Exploring the effect of color on cognitive task performances. Science 323:1226-1229

46. Cooper BA, Ward M, Gowland CA et al (1991) The use of the Lanthony New Color test in determining the effects of aging on color vision. J Gerontol 46:320-324

47. Nightingale F (1859) Notes on Nursing. Harrison and Sons, St. Martin's Lane, W.C.

48. Shikder S, Mourshed M, Price A (2012) Therapeutic lighting design for the elderly: a review. Perspect Pub Health 132:282-291

49. Kuijsters A, Redi J, de Ruyter B et al (2015) Lighting to make you feel better: Improving the mood of elderly people with affective ambiences. PLoS One 20;10:e0132732

50. Fontana Gasio P, Kräuchi K, Cajochen C et al (2003) Dawndusk simulation light therapy of disturbed circadian rest-activity cycles in demented elderly. Exp Gerontol 38:207-216

51. Kobayashi R, Fukuda N, Kohsaka M et al (2001) Effects of bright light at lunchtime on sleep of patients in a geriatric hospital. Psychiatry Clin Neurosci 55:287-289

52. Terman M, Terman JS (2010) Light therapy. In: Kryger MH, Roth T, Dement WC (eds) Principles and practice of sleep medicine. Elsevier, New York

53. Terman M, Terman JS (2005) Light therapy for seasonal and nonseasonal depression: efficacy, protocol, safety, and side effects. CNS Spectr 10:647-663

54. Golden RN, Gaynes BN, Ekstrom RD et al (2005) The efficacy of light therapy in the treatment of mood disorders: a review and meta-analysis of the evidence. Am J Psychiatry 162:656-662

55. Ancoli-Israel S, Klauber MR, Jones DW et al (1997) Variations in circadian rhythms of activity, sleep, and light exposure related to dementia in nursing-home patients. Sleep 20:18-23

56. van Hoof J, Aarts MPJ, Rense CG et al (2009) Ambient bright light in dementia: effects on behavior and circadian rhythmicity. Build Environ 44:146-155

57. Vandewalle G, Collignon O, Hull JT et al (2013) Blue light stimulates cognitive brain activity in visually blind individuals. J Cogn Neurosci 25:2072-2085

58. Sloane PD, Figueiro M, Cohen L (2008) Light as therapy for sleep disorders and depression in older adults. Clin Geriatr $16: 25-31$

59. Holzman DC (2010) What's in a color? The unique human health effects of blue light. Environ Health Perspect 118:A22-A27 
60. Daneault V, Hebert M, Albouy G et al (2014) Aging reduces the stimulating effect of blue light on cognitive brain functions. Sleep 37:85-96

61. Deneault V, Vandewalle G, Hebert M et al (2012) Does pupil constriction under blue and green monochromatic light exposure change with age? J Biol Rhythms 27:257-264

62. Artigas JM, Felipe A, Navea A et al (2012) Spectral transmission of the human crystalline lens in adult and elderly persons: color and total transmission of visible light. Investig Ophthalmol Vis Sci 53:4076-4084

63. Barbur JL, Konstantakopoulou E (2012) Changes in color vision with decreasing light level: separating the effects of normal aging from disease. J Opt Soc Am A 29:A27-A35

64. Lange JE, Morey JA, Richards OW (1980) Cool white, ultralume, and vita-lite fluorescent lamps for use in color vision testing. Am J Optom Physiol Opt 57:400-403

65. Veitch JA, McColl SL (2001) A critical examination of perceptual and cognitive effects attributed to full-spectrum fluorescent lighting. Ergonomics 44:255-279

66. Knez I (2014) Affective and cognitive reactions to subliminal flicker from fluorescent lighting. Conscious Cogn 26:97-104

67. Wakamura T, Tokura H (2001) Influence of bright light during daytime on sleep parameters in hospitalized elderly patients. J Appl Anthropol Appl Hum Sci 20:345-351

68. Taguchi T (2013) Bright light treatment for prevention of perioperative delirium in elderly patients. J Nurs Educ Pract 3:10-18

69. Park JY, Ha RY, Ryu E et al (2013) Effects of color temperature and brightness on electroencephalogram alpha activity in a polychromatic light-emitting diode. Clin Psychopharmacol Neurosci 11:126-131

70. Rahman SA, St. Hilaire MA, Lockley SW (2017) The effects of spectral tuning on evening ambient light on melatonin suppression, alertness and sleep. Physiol Behav 177:221-229

71. Philips Lighting (2018) 12T8/48-3000 specifications. http:// www.assets.lighting.philips.com/is/content/PhilipsLighting/ fp929001123904-pss-global. Accessed 15 May 2018

72. Philips Lighting (2018) 12T8/48-5000 specifications. http:// www.assets.lighting.philips.com/is/content/PhilipsLighting/ fp929001124204-pss-global. Accessed 15 May 2018

73. Dennis CM, Lee R, Woodard EK et al (2010) Benefits of quiet time for neuro-intensive care patients. J Neurosci Nurs 42:217-224

74. Engwall M, Fridh I, Johansson L et al (2015) Lighting, sleep and circadian rhythm: an intervention study in the intensive care unit. Intensive Crit Care Nurs 31:325-335

75. Figueiro MG, Hnter CM, Higgins P et al (2015) Tailored lighting intervention for persons with dementia and caregivers living at home. Sleep Health 1:322-330

76. Simons KS, Laheij RJ, van den Boogaard M et al (2016) Dynamic light application therapy to reduce the incidence and duration of delirium in intensive-care patients: a randomized controlled study. Lancet Respir Med 4:194-202

77. Engwall M, Fridh I, Jutengren G et al (2017) The effect of cycled lighting in the intensive care unit on sleep, activity and physiologic parameters: a pilot study. Intensive Crit Care Nurs 41:26-32

78. Dube JAO, Barth MM, Cmiel CA et al (2008) Environmental noise sources and interventions to minimize them: a tale of 2 hospitals. J Nurs Care Quality 23:216-224

79. Johansson L, Knutsson S, Bergbom I et al (2016) Noise in the ICU patient room-Staff knowledge and clinical improvements. Intensive Crit Care Nurs 35:1-9

80. Patel J, Baldwin J, Bunting P et al (2014) The effect of a multicomponent multidisciplinary bundle of interventions on sleep and delirium in medical and surgical intensive care patients. Anaesthesia 69:540-549
81. Izawa S, Kuzuya M, Okada K et al (2006) The nutritional status of frail elderly with care needs according to the mini-nutritional assessment. Clin Nutr 25:962-967

82. Guigoz Y (2006) The mini nutritional assessment (MNA): review of the literature-what does it tell us? J Nutr Health Aging 10:466-477

83. Johansson L, Sidenvall B, Malmberg B et al (2009) Who will become malnoursihed? A prospective study of factors associated with malnutrition in older persons living at home. J Nutr Health Aging 13:855-861

84. Johansson L, Bjorklund A, Sidenvall B et al(2015) Staff views on how to improve mealtimes for elderly people living at home. Dementia 16:835-852

85. McCormack P (1997) Undernutrition in the elderly population living at home in the community: a review of the literature. J Adv Nurs 26:856-863

86. Frangos E, Trombetti A, Graf CE et al (2016) Malnutrition in very old hospitalized patients: a new etiologic factor of anemia? J Nutr Health Aging 20:705-713

87. Bruyere O, Cavalier E, Buckinx F et al (2017) Relevance of vita$\min \mathrm{D}$ in the pathogenesis and therapy of frailty. Curr Opin Clin Nutr Metabol Care 20:26-29

88. Yannakoulia M, Ntanasi E, Anastasiou CA et al (2017) Frailty and nutrition: from epidemiological and clinical evidence to potential mechanisms. Metabol Clin Exp 68:64-76

89. Bhattacharya B, Maung A, Schuster K et al (2016) The older they are the harder they fall: injury patterns and outcomes by age after ground level falls. Injury 47:1955-1959

90. Winters B (2009) Older adults with traumatic rib fractures: an evidenced-based approach to their care. J Trauma Nurs 16:93-97

91. Sahr SM, Webb ML, Renner CH et al (2013) Implementation of a rib fracture triage protocol in elderly trauma patients. J Trauma Nurs 20:172-175

92. Pyke OJ, Rubano JA, Vosswinkel JA et al (2017) Admission of elderly blunt thoracic trauma patients directly to the intensive care unit improves outcomes. J Surg Res 219:334-340

93. Barry R, Thompson E (2018) Outcomes after rib fractures in geriatric blunt trauma patients. Am J Surg. https://doi.org/10.1016/j. amjsurg.2018.03.011.

94. Gary RA, Sueta CA, Dougherty M et al (2004) Home-based exercise improves functional performance and quality of ife in women with diastolic heart failure. Heart Lung 33:210-218

95. Middleton LE, Mitnitski A, Fallah N et al (2008) Changes in cognition and mortality in relation to exercise in late life: a population based study. PLoS One 3:e3124

96. Napoli N, Shah K, Waters DL et al (2014) Effect of weight loss, exercise, or both on cognition and quality of life in obese older adults. Am J Clin Nutr 100:189-198

97. Santanasto AJ, Glynn NW, Lovato LC et al (2017) Effect of physical activity versus health education on physical function, grip strength and mobility. J Am Geriatr Soc 65:1427-1433

98. Haider S, Dorner TE, Luger E et al (2017) Impact of a homebased physical and nutritional intervention program conducted by lay-volunteers on handgrip strength in prefrail and frail older adults: a randomized control trial. PLoS One 12:e0169613

99. Carpintero P, Caeiro JR, Carpintero R et al (2014) Complications of hip fractures: a review. World J Orthop 5:402-411

100. Tsuda Y, Yasunaga H, Horiguchi H et al (2015) Association between dementia and postoperative complications after hip fracture surgery in the elderly: analysis of 87,654 patients using a national administrative database. Arch Orthop Trauma Surg 135:1511-1517

101. Hassan M, Tuckman HP, Patrick RH et al (2010) Cost of hospital-acquired infection. Hosp Top 88:82-89

102. Bail K, Goss J, Draper B et al (2015) The cost of hospitalacquired complications for older people with and without 
dementia: a retrospective cohort study. BMC Health Serv Res 15:91

103. Rozanska A, Walaszek M, Wolak Z et al (2016) Prolonged hospitalization of patients with hospital acquired pneumoniae in the intensive care unit-morbidity, mortality, and costs of. Przegl Epidemiol 70:449-461

104. Foxamn B (2002) Epidemiology of urinary tract infections: Incidence, morbidity, and economic costs. Am J Med 113:5s-13s

105. Dimick JB, Chen SL, Taheri PA et al (2004) Hospital costs associated with surgical complications: a report from the privatesector Narional Surgical Quality Improvement Program. J Am Coll Surg 199:531-537
106. Vonlanthen R, Slankamenac K, Breitenstein S et al (2011) The impact of complications on costs of major surgical procedures. Ann Surg 254:907-913

107. Zywiel MG, Hurley RT, Perruccio AV et al (2015) Health economic implications of perioperative delirium in older patients after surgery for a fragility hip fracture. J Bone Joint Surg 97A:829-836

Publisher's Note Springer Nature remains neutral with regard to jurisdictional claims in published maps and institutional affiliations. 